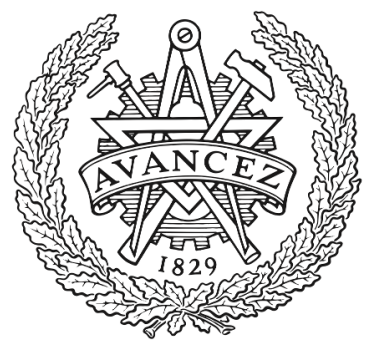

CHALMERS

UNIVERSITY OF TECHNOLOGY

\title{
Design of Programmable Filterless Optical Networks
}

Downloaded from: https://research.chalmers.se, 2023-04-26 09:40 UTC

Citation for the original published paper (version of record):

Furdek Prekratic, M., Natalino Da Silva, C., Wosinska, L. et al (2020). Design of Programmable Filterless Optical Networks. 2020 Photonics North, PN 2020.

http://dx.doi.org/10.1109/PN50013.2020.9166942

N.B. When citing this work, cite the original published paper. 


\section{Design of Programmable Filterless Optical Networks}

\author{
Marija Furdek, Carlos Natalino, Lena Wosinska \\ Dept. of Electrical Engineering \\ Chalmers University of Technology \\ Gothenburg, Sweden \\ furdek@chalmers.se
}

\begin{abstract}
We present the main operating principles and guidelines for the design of programmable filterless networks.

Keywords-filterless networks; programmable nodes; routing; spectrum assignment
\end{abstract}

\section{INTRODUCTION}

To satisfy the network traffic growth in an economically viable manner, telecom operators are seeking agile and costefficient optical networking solutions. Supreme flexibility in terms of nodal architecture and network provisioning is provided by programmable, or white box optical switches [1], where the optical components (e.g., wavelength-selective switches and splitters) are interconnected through a reconfigurable optical backplane (OB) (e.g., a piezoelectric switch). By (re)arranging the $\mathrm{OB}$, the node layout can be designed to tightly fit the processing requirements of individual connections.

On the opposite side of the agility-vs-cost gamut lie passive filterless optical networking solutions based on coherent transceivers interconnected only by passive splitters and combiners [2]. Due to the absence of filtering, signals follow Drop\&Continue transmission - they are broadcasted to all links in the fiber tree downstream of the source node and propagate along the links beyond the destination node. These networks provide innate support for gridless operation, are reliable, and cost- and energy-efficient [3]. Their benefits in the core and metro segment have already been demonstrated [4], leading to practical deployments. However, in addition to the rigidness of this architecture, the absence of filtering generates spectrum waste, demanding more flexible solutions.

\section{Programmable Filterless Networks}

Programmable filterless networks (PFNs) are a trade-off solution that combines the advantages of the programmable optical nodes and filterless transmission while offsetting their

\author{
Christine Tremblay \\ Dept. of Electrical Engineering \\ École de technologie supérieure, Université du Québec \\ Montréal, Canada
}

drawbacks [5]. Fig. 1 illustrates the nodal architecture in passive filterless, Reconfigurable Optical Add/Drop Multiplexer (ROADM)-based and programmable filterless networks supporting 5 connections. PFNs deploy white box switches with a programmable OB to provide the necessary interconnections between input and output ports of each node. This flexibility reduces the spectrum waste compared to the fully passive architecture because signals are split only when necessary (i.e., when multiple signals from the same input must be sent to different outputs).

Design of PFNs entails several important tasks. In addition to the standard Routing, Modulation Format and Spectrum Assignment (RMSA) problem of assigning spectral and spatial resources to connection demands, the nodal architecture that best supports them also needs to be determined. This requires defining the number, degree, and position of the passive couplers and optical amplifiers inside each node. In this talk, we will examine the optimization framework for RMSA and node architecture design in PFNs, and evaluate the advantages of this architecture through a comparison with ROADM-based and passive filterless networks.

\section{REFERENCES}

[1] N. Amaya et al., "Introducing node architecture flexibility for elastic optical networks," IEEE/OSA J. Opt. Commun. Netw., vol. 5, no. 6, pp. 593-608, June 2013.

[2] C. Tremblay et al., "Filterless optical networks: A unique and novel passive wan network solution," in OECC/IOOC, 2007.

[3] E. Archambault et al., "Routing and spectrum assignment in elastic filterless optical networks," IEEE/ACM Trans. Netw., vol. 24, no. 6, pp. 3578-3592, Dec. 2016.

[4] P. Pavon-Marino et al., "Techno-economic impact of filterless data plane and agile control plane in the $5 \mathrm{G}$ optical metro," IEEE/OSA J. Lightwave Techn., early access, DOI 10.1109/JLT.2020.2982131.

[5] M. Furdek et al., "Programmable filterless network architecture based on optical white boxes," in ONDM, 2016.

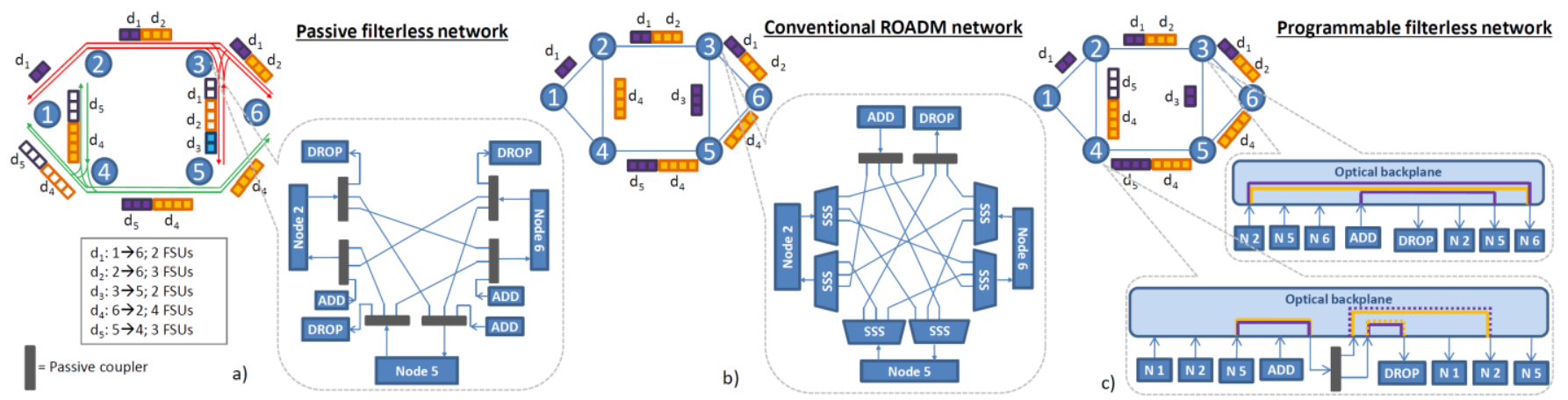

Figure 1. An illustrative example of a) passive filterless, b) conventional ROADM-based, and c) programmable filterless network architecture supporting five connection requests, along with the configuration of representative nodes. 